\title{
MRI index lesion radiomics and machine learning for detection of extraprostatic extension of disease: a multicenter study
}

\author{
Renato Cuocolo ${ }^{1,2}$ - Arnaldo Stanzione ${ }^{3} \cdot$ Riccardo Faletti $^{4} \cdot$ Marco Gatti $^{4}$ (D) $\cdot$ Giorgio Calleris $^{5}$ - Alberto Fornari $^{6}$. \\ Francesco Gentile ${ }^{4}$. Aurelio Motta ${ }^{4}$. Serena Dell'Aversana ${ }^{3} \cdot$ Massimiliano Creta $^{7}$ - Nicola Longo ${ }^{7}$. Paolo Gontero ${ }^{5}$. \\ Stefano Cirillo ${ }^{6} \cdot$ Paolo Fonio $^{4} \cdot$ Massimo $^{4}$ Imbriaco $^{3}$
}

Received: 22 October 2020 / Revised: 23 January 2021 / Accepted: 9 March 2021 / Published online: 1 April 2021

(C) The Author(s) 2021

\begin{abstract}
Objectives To build a machine learning (ML) model to detect extraprostatic extension (EPE) of prostate cancer (PCa), based on radiomics features extracted from prostate MRI index lesions.

Methods Consecutive MRI exams of patients undergoing radical prostatectomy for PCa were retrospectively collected from three institutions. Axial T2-weighted and apparent diffusion coefficient map images were annotated to obtain index lesion volumes of interest for radiomics feature extraction. Data from one institution was used for training, feature selection (using reproducibility, variance and pairwise correlation analyses, and a correlation-based subset evaluator), and tuning of a support vector machine (SVM) algorithm, with stratified 10-fold cross-validation. The model was tested on the two remaining institutions' data and compared with a baseline reference and expert radiologist assessment of EPE.

Results In total, 193 patients were included. From an initial dataset of 2436 features, 2287 were excluded due to either poor stability, low variance, or high collinearity. Among the remaining, 14 features were used to train the ML model, which reached an overall accuracy of $83 \%$ in the training set. In the two external test sets, the SVM achieved an accuracy of $79 \%$ and $74 \%$ respectively, not statistically different from that of the radiologist $(81-83 \%, p=0.39-1)$ and outperforming the baseline reference $(p=0.001-0.02)$.

Conclusions A ML model solely based on radiomics features demonstrated high accuracy for EPE detection and good generalizability in a multicenter setting. Paired to qualitative EPE assessment, this approach could aid radiologists in this challenging task.

Key Points

- Predicting the presence of EPE in prostate cancer patients is a challenging task for radiologists.

- A support vector machine algorithm achieved high diagnostic accuracy for EPE detection, with good generalizability when tested on multiple external datasets.

- The performance of the algorithm was not significantly different from that of an experienced radiologist.
\end{abstract}

Keywords Magnetic resonance imaging $\cdot$ Machine learning $\cdot$ Support vector machine $\cdot$ Prostate cancer $\cdot$ Prostatectomy

Renato Cuocolo and Arnaldo Stanzione contributed equally to the study and should be considered co-first authors.

Marco Gatti

marcogatti17@gmail.com

1 Department of Clinical Medicine and Surgery, University of Naples "Federico II", Naples, Italy

2 Laboratory of Augmented Reality for Health Monitoring (ARHeMLab), Department of Electrical Engineering and Information Technology, University of Naples "Federico II", Naples, Italy

3 Department of Advanced Biomedical Sciences, University of Naples "Federico II", Naples, Italy
4 Department of Surgical Sciences, Radiology Unit, University of Turin, Via Genova 3, 10126 Turin, Italy

5 Division of Urology, Città della Salute e della Scienza, Molinette Hospital, University of Turin, Torino, Italy

6 Radiology Unit, Mauriziano Umberto I Hospital, 10128 Turin, Italy

7 Department of Neurosciences, Reproductive Sciences and Odontostomatology, University of Naples "Federico II", Naples, Italy 


\begin{tabular}{|c|c|}
\hline \multicolumn{2}{|c|}{ Abbreviations } \\
\hline ADC & Apparent diffusion coefficient \\
\hline AUC & $\begin{array}{l}\text { Area under the Receiver Operating Characteristics } \\
\text { curve }\end{array}$ \\
\hline AUPRC & Area under the Precision-Recall curve \\
\hline DWI & Diffusion-weighted images \\
\hline EPE & Extraprostatic extension of disease \\
\hline ICC & Intercorrelation coefficient \\
\hline ISUP & International Society of Urological Pathology \\
\hline $\mathrm{MCC}$ & Matthew's correlation coefficient \\
\hline mpMRI & Multiparametric MRI \\
\hline $\mathrm{PCa}$ & Prostate cancer \\
\hline $\begin{array}{l}\text { P I - } \\
\text { RADS }\end{array}$ & Prostate Imaging and Reporting Data System \\
\hline PSA & Prostate-specific antigen \\
\hline $\mathrm{ROC}$ & Receiver operating characteristics \\
\hline SVM & Support vector machine \\
\hline $\mathrm{T} 2 \mathrm{w}$ & T2-weighted \\
\hline
\end{tabular}

\section{Introduction}

The diagnostic pathway for prostate cancer (PCa) is rapidly evolving, with multiparametric MRI (mpMRI) gaining an increasingly central role in tumor detection. Indeed, it allows to identify lesions worthy of targeted biopsies, which when paired to systematic sampling leads to a more accurate and clinically relevant $\mathrm{PCa}$ assessment [1-3]. However, the value of mpMRI could go beyond PCa detection, and a great attention is presently paid to its accuracy in the identification of extraprostatic extension of disease (EPE) [4]. While confirmed organ-confined disease at mpMRI could lead to more conservative surgical approaches, mpMRI suffers from a relatively low and heterogeneous sensitivity that currently prevents its widespread adoption for this task $[5,6]$. This could be at least in part due to a lack of standardization in the interpretation of the multiple EPE signs detectable at mpMRI, although inherent limitations of the technique cannot be excluded. In this light, strategies to increase the performance of mpMRI for PCa local staging have been recently investigated and dedicated scoring systems for the reporting of EPE on mpMRI have been released, with the one named EPE grade proposed by Mehralivand et al that appears to be the most promising and awaiting validation [7-10]. Among the several mpMRI features suspicious for EPE, this scoring system focuses on tumor capsular contact length, capsular bulge, and/or irregularity and frank capsular breach [9]. However, there are other recognized mpMRI signs suggested by the latest release of Prostate Imaging and Reporting Data System (PI-RADS v2.1) that radiologists should consider in their decision making regarding EPE prediction, such as asymmetry of the neurovascular bundles and obliteration of the rectoprostatic angle [11]. Furthermore, the optimal tumor capsular contact threshold for EPE prediction still needs to be defined with EPE grade and PI-RADS v2.1 suggesting different cut-offs [9, 11-13]. Concurrently, prostate mpMRI has been one of the many imaging modalities' object of study in the field of radiomics, which is a multi-step process allowing the extraction of quantitative features from medical images that can be used to build decision support models [14-16]. Exploratory radiomics studies have been performed to assess the feasibility of applying radiomics, in combination with machine learning (ML) or not, for EPE prediction on mpMRI images, with encouraging results [17-21]. Nevertheless, their findings were limited in terms of generalizability due to the adoption of single-center datasets. With the present work, we aimed to evaluate the performance of a ML algorithm powered by radiomics data alone in the identification of EPE and compare its diagnostic accuracy to that of expert radiologists, using two independent external datasets for validation.

\section{Materials and methods}

The respective Local Institutional Review Board for each Institution approved this retrospective study and waived the need for written informed consent.

\section{Patient population}

This study was conducted enrolling patients from Molinette Hospital, Turin (site 1); Mauriziano Umberto I Hospital, Turin (site 2); and Federico II Hospital, Naples (site 3), Italy. We retrospectively reviewed consecutive patients who underwent prostate MRI at each site for $\mathrm{PCa}$ suspicion between November 2015 and October 2018. Inclusion criteria were the following: presence of an index lesion (PI-RADS score $\geq 3$, defined according to PI-RADS guidelines, assigned by the original reader) with bioptic confirmation of $\mathrm{PCa}$ presence (defined as Gleason score $\geq 3+3$ ) in the index lesion through targeted biopsy within 3 months of MRI; treatment with RP within 3 months of biopsy. Only patients with significant artifacts at MRI or incomplete exams (i.e., interrupted for claustrophobia) were excluded from subsequent analyses. Pathology reports were analyzed to assess if EPE was identified on RP specimens at the location of the index lesion, based on the International Society of Urological Pathology consensus conference criteria [22]. Patients from site 1 were used to train and tune the ML model while those from sites 2 and 3 were employed as distinct external test sets to validate its performance. 


\section{MRI acquisition}

MRI examinations at sites 1 and 2 were performed on 1.5-T scanners (Achieva and Ingenia, Philips Medical Systems). A 3-T scanner was employed in site 3 (Magnetom Trio, Siemens Medical Solutions), using surface phase-arrayed and integrated spine phased-array coils. None of the sites employed endorectal coils. All acquisition protocols included axial T2-weighted (T2w) and diffusion-weighted images (DWI), with corresponding apparent diffusion coefficient maps (ADC). Further details are available in the supplementary materials.

\section{Radiomics analysis}

All axial T2w and ADC images from included patients were manually anonymized and converted to the NIfTI format prior to analysis, using dcm2niix [23]. Index lesion location was provided to a genitourinary radiologist ( $>5$ years of experience), who performed a manual segmentation of the entire index lesion volume on both $\mathrm{T} 2 \mathrm{w}$ and ADC images (Fig. 1). To assess the feature reproducibility in relation to manual segmentation, two other readers (a radiologist and a radiology resident) independently annotated a subset of 30 randomly selected patients from the site 1 training set. The supplementary materials contain further details on the additional annotations. These segmentations were used for intercorrelation coefficient (ICC) calculation in one of the feature selection steps as detailed below. Dedicated software was used for all segmentations (ITK-SNAP, v3.8) [24].

PyRadiomics (v3.0) was employed for feature extraction [25]. Preprocessing was performed with voxel resampling to $1 \times 1 \times 1 \mathrm{~mm}$, whole-image gray level $z$-score normalization, scaling by a 100 factor and array shift (by 300), followed by discretization with a fixed bin width $(=5)$. Both original and filtered images were used to calculate 3D shape, first order, and texture features. In detail, the Laplacian of Gaussian filtering with multiple sigma values $(=1,2,3,4,5)$ and wavelet decomposition with all combinations of high- and low-pass filters in the $x$-, $y$-, and $z$-axes were employed to highlight textural characteristics of the index lesion. The supplementary materials contain the complete settings file used for the extraction.

\section{Feature preprocessing and selection}

The scikit-learn Python3 package and the Weka data mining software were used for the subsequent steps of the analysis (v3.8) $[26,27]$. The feature selection process was conducted exclusively on the site 1 training dataset, to avoid any information leak which could bias the final model. A normalization scaler (range $=0-1$ ) was fit on the training data and used to transform all 3 datasets.

The first step of feature selection consisted in the exclusion of non-reproducible features through ICC analysis of the
Fig. 1 Prostate MR images (axial T2-weighted on the left and ADC map on the right) from a 76-yearold patient with a PI-RADS 5 transition zone lesion involving the anterior fibromuscular stroma (Gleason score $4+3$ and signs of extraprostatic extension of disease at prostatectomy). The slices in which the lesion was more conspicuous are shown respectively before (a and $\mathbf{b}$ ) and after (c and d) after manual segmentation
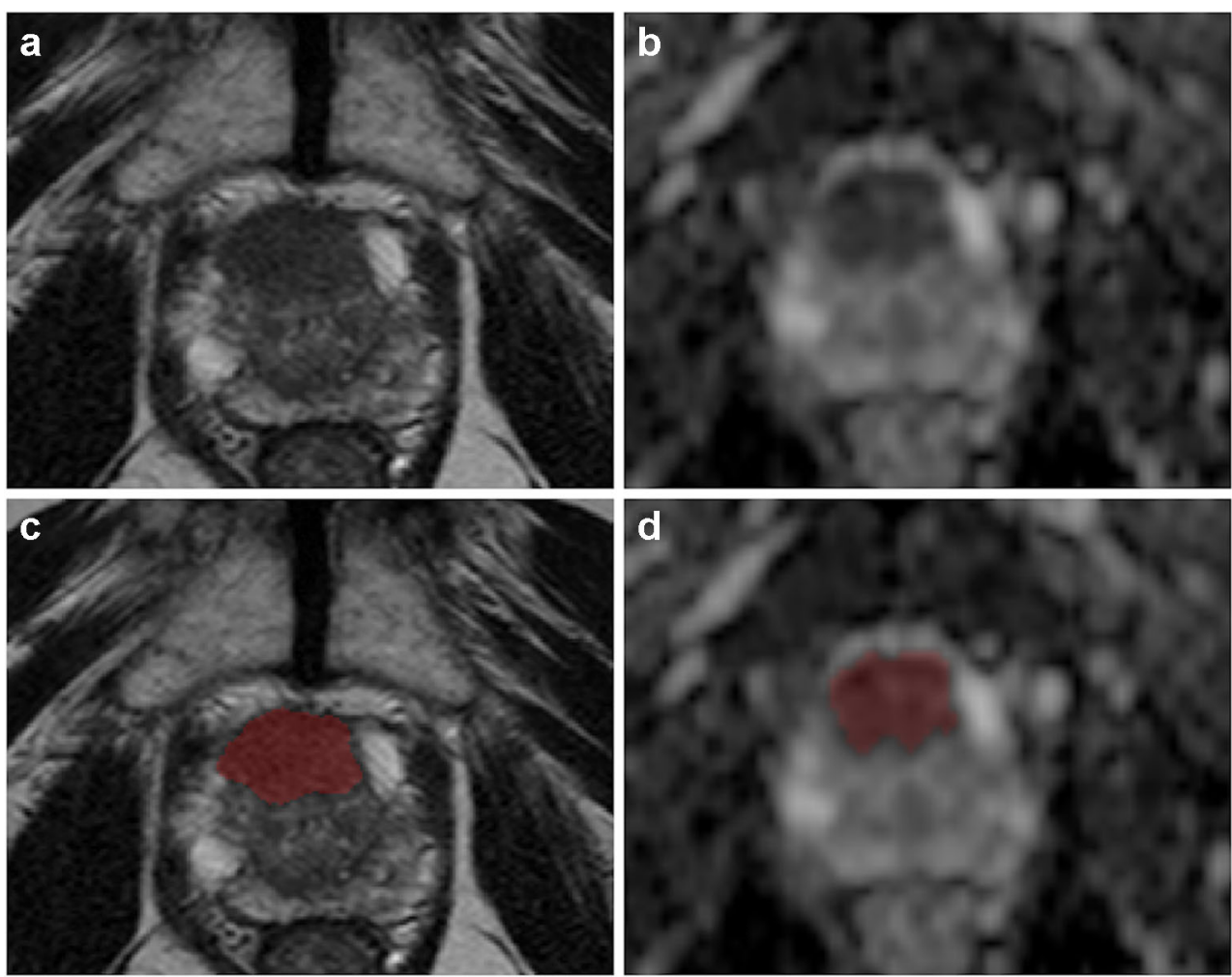
results obtained by the three independent readers. Radiomics features were extracted, using the same settings, from their respective annotation sets $(n=30)$. The resulting datasets were used to calculate the ICC value of each parameter. A two-way random effect, single rater, absolute agreement ICC model was employed, and a value $\geq 0.75$ was considered "good reproducibility," the minimum requirement for inclusion in the analysis [28]. Subsequently, a variance filter (threshold = 0.1 ) was applied to each feature to remove parameters with low information content. Using pairwise correlation, features with high collinearity (threshold $>0.8$ ) were also excluded from the analysis. Finally, the Weka data mining platform (v3.9) correlation-based feature subset evaluator was used to identify the best feature subset among the remaining.

\section{Machine learning}

A support vector machine (SVM) algorithm was employed to develop a predictive model for EPE. Training set classes were balanced using the Synthetic Minority Oversampling Technique [29]. A stratified 10-fold cross-validation was used for model tuning prior to final training on the entire site 1 dataset. The final model performance was then independently tested on 2 external datasets (sites 2 and 3) calculating confusion matrix-derived accuracy metrics and receiver operating characteristics (ROC) curves. Brier score and calibration curves were also obtained for each test set to evaluate prediction and calibration loss. For each center included in the study, an expert radiologist (all $>5$ years of experience in prostate MRI) performed an assessment based on the entire prostate mpMRI exam and each case was classified as positive or negative for EPE using previously established signs from the PI-RADSv2.1 guidelines [30]. No PI-RADS scores were assigned during these readings since PI-RADS scores were not included in the ML analysis. These readings were used to provide a comparison for the SVM's performance.
An overview of the complete analysis pipeline is presented in Fig. 2.

\section{Statistical analysis}

Continuous variables were tested for normality using the Shapiro-Wilk test and are presented as mean and standard deviation or median and interquartile range accordingly. Ordinal data are presented as value counts, categorical data as proportions. The Kruskal-Wallis rank sum test was used to assess differences in clinical data among the datasets, with a Dunn test post hoc analysis if necessary. A Fisher exact test was used to compare the distribution of EPE cases between the 3 sites. Model accuracy ( $n$ correct predictions/total cases) in each test set was also compared with a baseline reference (no information rate, i.e., the class mode) using a binomial test and an expert radiologist's predictions using McNemar's tests. A $p<0.05$ was considered statistically significant, with correction for multiple comparisons when required. The statistical analysis was performed using the R software environment [31].

\section{Results}

In total, 193 patients met the selection criteria: 104 from site 1, 43 from site 2, and 46 from Site 3. Their clinical and demographic data are reported in Table 1 . No significant differences were found among the three populations, with the exception of PI-RADS scores of Sites 2 and $3(p=0.008)$. From their exams, 1218 radiomics features were extracted from $\mathrm{T} 2 \mathrm{w}$ and ADC images (complete dataset $=2436$ features). Of these, $55 \%(n=675 / 1218)$ from T2w images and 73\% $(n=892 /$ 1218) from ADC maps resulted not reproducible. Among the remaining 869 parameters, 21 (2\%) presented low variance and were also removed from the training dataset. The intercorrelation analysis led to the exclusion of an additional 699/
Fig. 2 Image analysis and machine learning pipeline

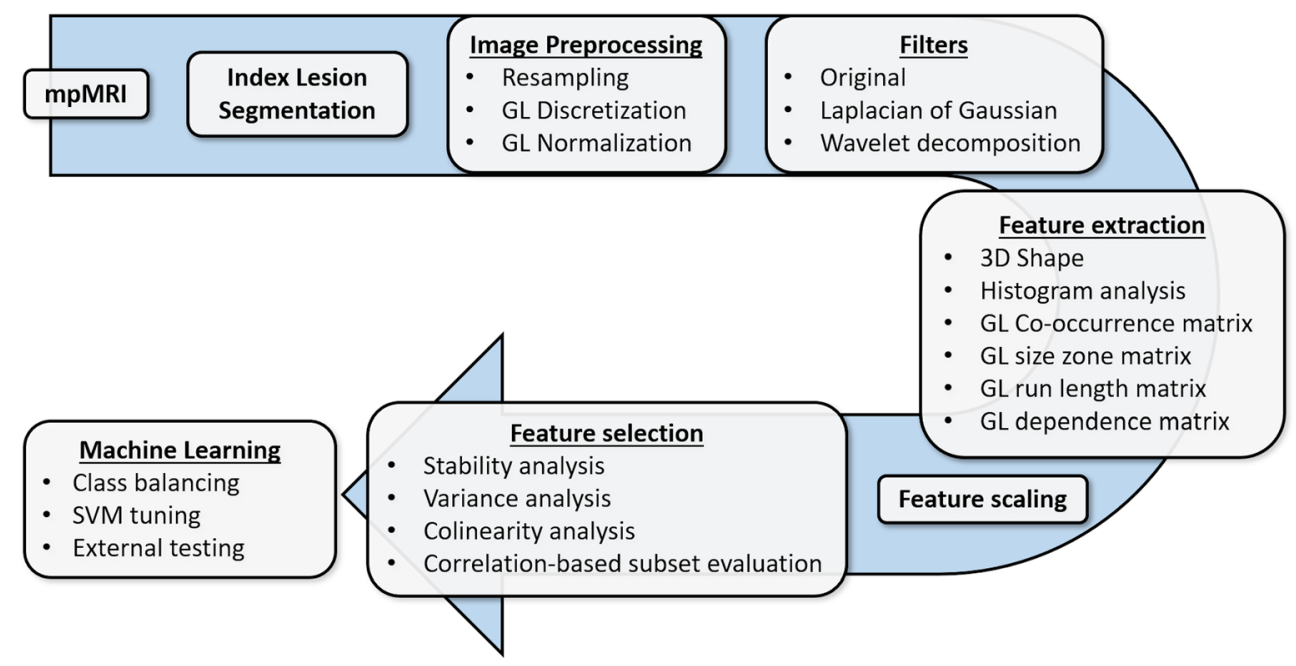


Table 1 Patient population clinical and demographic characteristics. Continuous data are presented as a median and interquartile range, ordinal data as value counts, and categorical data as proportions

\begin{tabular}{|c|c|c|c|c|}
\hline & Site 1 & Site 2 & Site 3 & \\
\hline Age (years) & $66(60-72)$ & $67(60-69)$ & $67(63-71)$ & $p=0.32$ \\
\hline PSA (ng/ml) & $7.1(5.12-10.00)$ & $6.93(5.51-9.78)$ & $8.00(5.35-9.76)$ & $p=0.89$ \\
\hline \multirow[t]{4}{*}{ ISUP $\operatorname{grade}^{\S}$} & $\begin{array}{l}1=1 \\
2=40\end{array}$ & $\begin{array}{l}1=1 \\
2=21\end{array}$ & $\begin{array}{l}1=5 \\
2=15\end{array}$ & \multirow[t]{4}{*}{$p=0.21$} \\
\hline & $3=43$ & $3=16$ & $3=8$ & \\
\hline & $4=17$ & $4=4$ & $4=14$ & \\
\hline & $5=3$ & $5=1$ & $5=5$ & \\
\hline \multirow[t]{2}{*}{ PI-RADS score } & $\begin{array}{l}3=3 \\
4=68\end{array}$ & $\begin{array}{l}3=7 \\
4=24\end{array}$ & $\begin{array}{l}3=3 \\
4=19\end{array}$ & \multirow[t]{2}{*}{$p=0.02 *$} \\
\hline & $5=33$ & $5=12$ & $5=25$ & \\
\hline EPE (pathologically proven) & $37 / 104$ & $19 / 43$ & $20 / 47$ & $p=0.55$ \\
\hline
\end{tabular}

$P S A$, prostate-specific antigen; ISUP, International Society of Urological Pathology; PI-RADS, Prostate Imaging and Reporting Data System; EPE, extraprostatic extension of disease

*Post hoc analysis showed a significant difference exclusively between sites 2 and $3(p=0.008)$

$\S$ As originally assigned by the pathologist on the radical prostatectomy specimen

\# Obtained from the original radiology report

848 features (82\%) and the correlation-based subset evaluator identified 14 features to be employed in training the model, listed in the supplementary materials (Figs. 3 and 4).

The final model was an SVM classifier with a radial basis function kernel, $C=3$ and gamma $=0.01$; the trained model file is available in the supplementary materials. In the training set, an overall accuracy (correct/overall instances) of $83 \%$ was obtained, with a 0.83 area under the ROC curve (AUC). In the external test sets, the SVM reached an accuracy of 79\% and AUC of 0.80 in the data from site 2 and $74 \%$ and 0.73 in site 3 (Fig. 5). The Brier score was 0.20 and 0.21 for sites 2 and 3 data, respectively (Fig. 6). In both test sets, ML outperformed the baseline reference ( $p=$ 0.001 in site $2, p=0.02$ in site 3 ). Confusion matrices and complete accuracy metrics are reported in Tables 2 and 3, respectively. The radiologist achieved an accuracy of $81 \%$ and $83 \%$ respectively in sites 2 and 3 . Both did not reach statistical significance when compared to ML ( $p=1$ for site 2 and $p=0.39$ for site 3) (Table 4). The confusion matrices for the radiologist's assessment are presented in the supplementary materials.

\section{Discussion}

Our study demonstrates the potential of radiomics-powered ML for the detection of EPE in PCa, which in turn could improve patient management and treatment choice. There
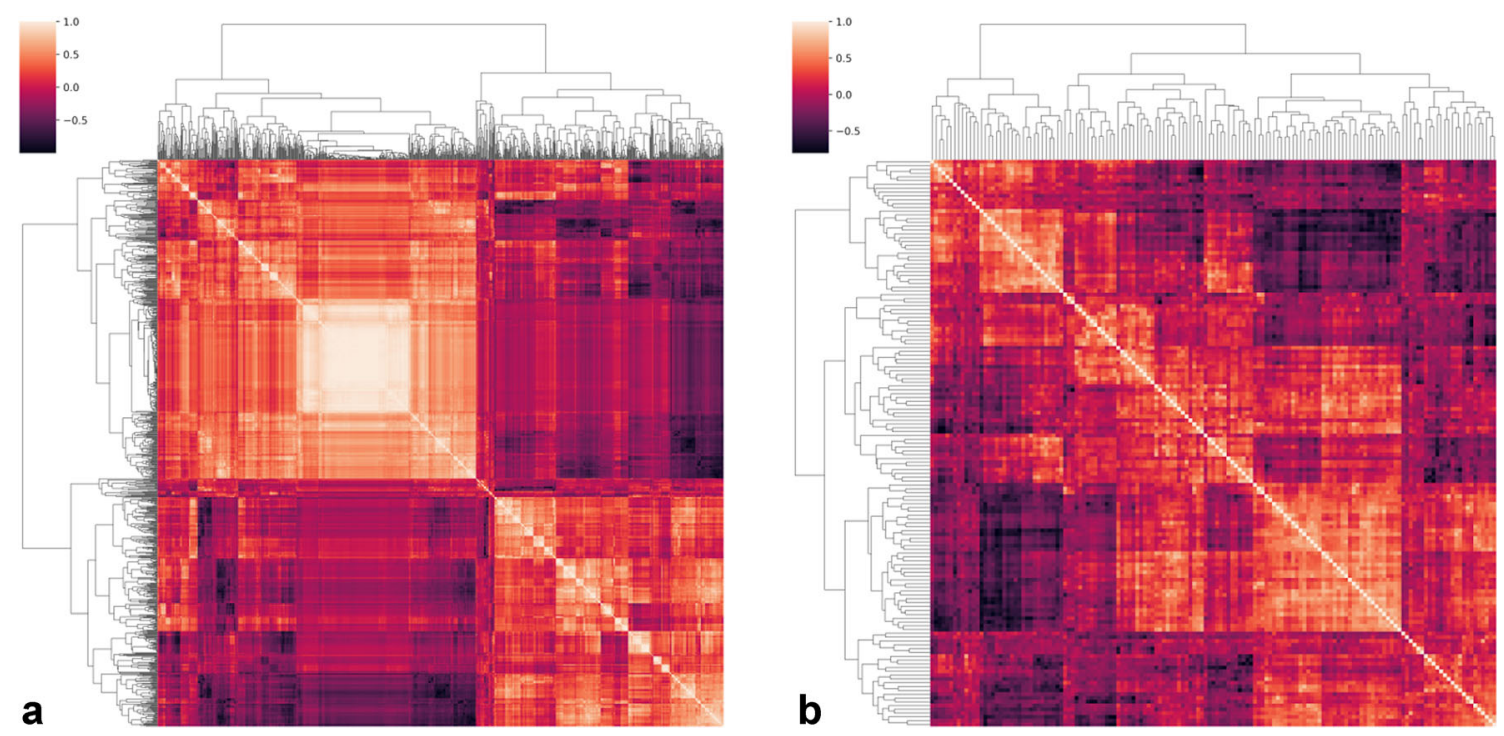

Fig. 3 Hierarchically clustered heatmap of feature pairwise correlation before (a) and after (b) removal of highly colinear ones 


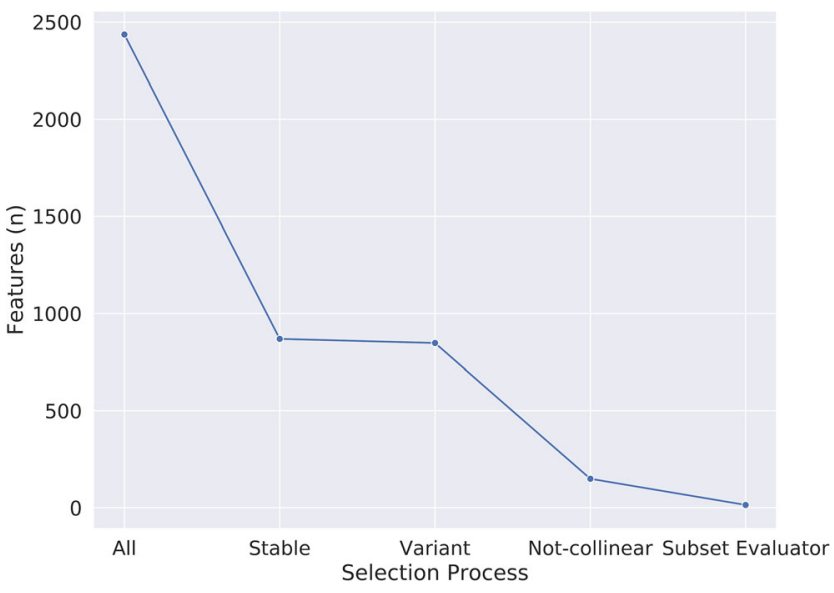

Fig. 4 Plot depicting parameter number (y-axis) reduction during the various feature selection steps ( $x$-axis)

have been several prior investigations that have focused on this task using a similar approach [17-21]. In our previous, single-center exploratory study of radiomics and ML for EPE detection, the accuracy achieved was similar (82\%), although only obtained through cross-validation in a single dataset [21]. In a prior investigation, Krishna et al had identified ADC map first-order features, in particular entropy, as promising EPE biomarkers (AUC 0.76) [19]. More recently, Ma et al obtained an $83 \%$ accuracy in a single institution 3T dataset, using 67 patients for testing the model [20]. Interestingly, in their study, they reported that the radiomics approach significantly outperformed the radiologist in EPE assessment, but exclusively in terms of sensitivity and not specificity or overall accuracy. Losnegard et al analyzed data acquired on a 1.5-T scanner using an endorectal coil, and their random forest model achieved an AUC of 0.74 [17]. This was again very similar to that of the radiologist $(0.75)$ on the same data. Finally, $\mathrm{Xu}$ et al were able to obtain an $82 \%$ accuracy on their test set, using data acquired on a 3-T scanner [18]. Our results in the training dataset are essentially in line with these previous

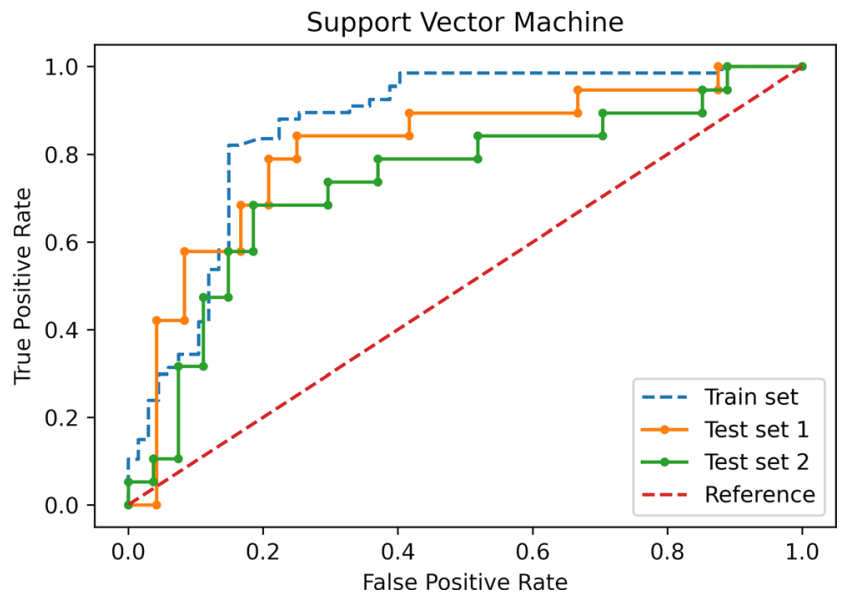

Fig. 5 Receiver operating characteristic curves of the support vector machine model in the train data and both test sets
Calibration Curve

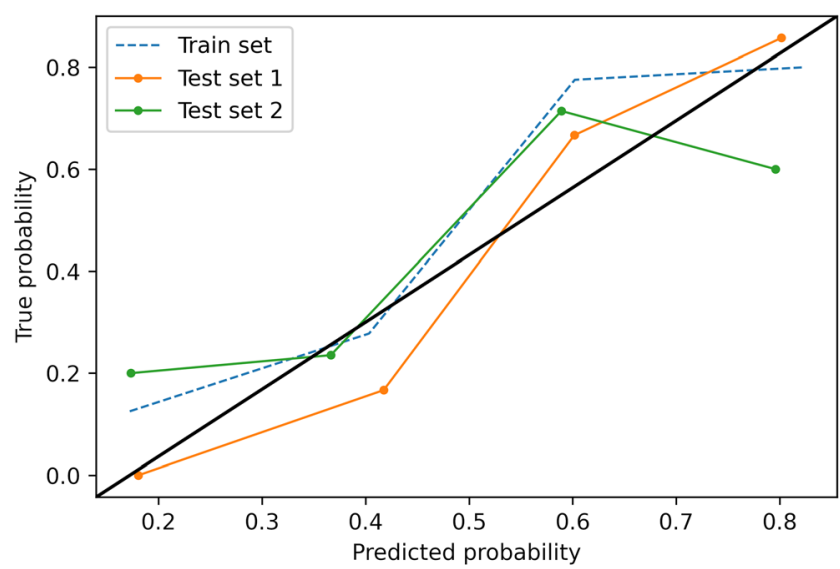

Fig. 6 Calibration curves of the support vector machine model in the train data and both test sets

studies ( $83 \%$ accuracy; however, none of these has performed external testing.

In the present investigation, we decided not to employ dynamic contrast-enhanced images (DCE) to obtain radiomics data on which to build our predictive model. This choice was dictated by several considerations, mainly the concern to ensure the widest possible applicability and generalizability of the resulting model as well as reducing sources of variability in our data as much as reasonably possible. Regarding the first, biparametric prostate MRI protocols without the use of DCE are becoming more and more common, also to accommodate the increasing demand for MRI exams due to its growing role in current guidelines [5, 32]. Protocols without DCE have also shown a similar performance to full mpMRI for EPE detection [33]. Finally, DCE has a high temporal resolution, with the degree and speed of lesion enhancement influenced both by technical and physiological factors, together with lesion nature. Therefore, it would be challenging to exclude all sources of bias from DCE radiomics features, potentially adding more noise to the data. Therefore, in the interest of keeping the model as simple as possible and employable in

Table 2 Confusion matrices for the SVM model

\begin{tabular}{llll}
\hline Sites & & Ground truth & \\
\cline { 3 - 4 } & & No EPE & EPE \\
\hline Site 2 & & & \\
SVM & No EPE & 18 & 3 \\
Site 3 & EPE & 6 & 16 \\
SVM & No EPE & 21 & 6 \\
& EPE & 6 & 13 \\
\hline
\end{tabular}

$S V M$, support vector machine; $E P E$, extraprostatic extension of disease 
Table 3 Accuracy metrics for the SVM model for the training (site 1) and testing (site 2 and site 3 ) datasets

\begin{tabular}{lllllll}
\hline & Sensitivity & $\begin{array}{l}\text { Positive } \\
\text { predictive value }\end{array}$ & $\begin{array}{l}\text { F- } \\
\text { measure }\end{array}$ & MCC & AUC & AUPRC \\
\hline Site 1 & & & & & \\
Absence of EPE & 0.78 & 0.87 & 0.82 & 0.66 & 0.83 & 0.79 \\
Presence of EPE & 0.88 & 0.80 & 0.84 & 0.66 & 0.83 & 0.76 \\
Weighted Average & 0.83 & 0.83 & 0.83 & 0.66 & 0.83 & 0.77 \\
Site 2 & & & & & & \\
Absence of EPE & 0.75 & 0.86 & 0.80 & 0.59 & 0.80 & 0.78 \\
Presence of EPE & 0.84 & 0.73 & 0.78 & 0.59 & 0.80 & 0.68 \\
Weighted Average & 0.79 & 0.80 & 0.79 & 0.59 & 0.80 & 0.74 \\
Site 3 & & & & & & \\
Absence of EPE & 0.78 & 0.78 & 0.78 & 0.46 & 0.73 & 0.74 \\
Presence of EPE & 0.68 & 0.68 & 0.68 & 0.46 & 0.73 & 0.60 \\
Weighted Average & 0.74 & 0.74 & 0.74 & 0.46 & 0.73 & 0.68 \\
\hline
\end{tabular}

$E P E$, extraprostatic extension of disease; $M C C$, Matthew's correlation coefficient; $A U C$, area under the receiver operating characteristics curve; $A U P R C$, area under the precision-recall curve most clinical settings, we decided to only focus on $\mathrm{T} 2 \mathrm{w}$ and ADC features for EPE predictive modeling.

As reproducibility of results represents one of the main limitations of both radiomics and ML, we chose a multicentric design to have the possibility to directly assess this issue by testing our model's results generalizability $[34,35]$. It should also be noted that our external test sets were constituted by exams acquired on different scanners with varying field strength compared to the training data. Our intention was to offer a better representation of real-world clinical practice and a better estimation of our model's performance. The results are very promising, with an accuracy of $83 \%$ in the training and $79 \%$ and $74 \%$ in the test sets and a performance comparable to that of an expert radiologist in both cases ( $p=1.00$ and 0.39 , respectively). It can be assumed that image preprocessing paired with robust feature selection, including feature stability testing, has reduced overfitting on noisy data. However, it should be noted that performance on the site 3 test set was still somewhat lower $(74 \%)$ than on site $2(79 \%)$. A bias due to

Table 4 Comparison of the SVM model and radiologist performances for the McNemar test

\begin{tabular}{llll}
\hline Sites & & \multicolumn{2}{l}{ SVM } \\
\cline { 3 - 4 } & & Error & Correct \\
\hline Site 2 & & & \\
Radiologist & Error & 4 & 4 \\
Site 3 & Correct & 5 & 30 \\
Radiologist & Error & 4 & 4 \\
& Correct & 8 & 30 \\
\hline
\end{tabular}

SVM, support vector machine case sampling cannot be completely excluded; i.e., more challenging cases were randomly present in one test set compared to the other, and this could be supported by the comparison with the radiologist's performance on the same data. On the other hand, we wish to highlight that site 3 had the greatest difference in terms of MRI scanner from site 1 , as it had both another vendor and higher field strength. Both these factors could also have contributed in varying degrees to the difference in model performance. Overall, the accuracy on the site 3 external test set can still be considered satisfactory, especially taking into account that it was still not significantly different from that of an expert radiologist ( $p=0.39$ ).

It is interesting to note that the AUCs obtained in the external test sets by our model $(0.73-0.80)$ are not far from those reported for experienced radiologists interpreting MR images using the EPE grade (0.77-0.81) [9, 10]. The EPE grade has shown a substantial inter- and intra-reader agreement and appears relatively simple to implement being based on relatively few imaging features [10]. However, it is still awaiting prospective validation and requires a certain degree of expertise to be used. It also does not solve the current limitations of mpMRI for EPE detection [36]. Our model exclusively requires lesion segmentation (a step that could also be automated) and would be easy to implement. It can be hypothesized that including our radiomics signature in the EPE grade scoring system might possibly further increase its diagnostic accuracy and reliability while supporting less experienced readers in the EPE assessment. On a similar note, future investigations could assess whether the inclusion of clinical and laboratory data, such as patient age, PSA/PSA-derived biomarkers, or biopsy Gleason score, may further improve our results.

Our study has some limitations that should be acknowledged. Its design was retrospective, which did not allow us to investigate more possible sources of limited radiomics 
feature reproducibility (e.g., scanner differences) in addition to manual segmentation. However, we believe a retrospective multicenter study is a necessary step after single-center experiences and prior to prospective clinical trials. We had an experienced radiologist from each study center assess the center's exams, which could determine some bias due to differences in performance. However, this choice was in our estimation better than having a single radiologist read exams acquired on MRI scanners with which he/she may not have been familiar, which could also have negatively influenced the outcome.

In conclusion, the combination of radiomics and ML has confirmed their promising performance for PCa EPE detection even in a multicenter setting. This tool could aid in improving patient management and be a valid support for radiologists in PCa staging. The next step in its development should be a prospective clinical trial.

Supplementary Information The online version contains supplementary material available at https://doi.org/10.1007/s00330-021-07856-3.

Acknowledgements Open access funding provided by Università degli Studi di Torino within the CRUI-CARE Agreement.

Funding The authors state that this work has not received any funding.

\section{Compliance with ethical standards}

Guarantor The scientific guarantors of this publication are Prof. Massimo Imbriaco and Prof. Paolo Fonio, chiefs of Diagnostic and Interventional Radiology at the University of Naples and Turin, respectively.

Conflict of interest The authors of this manuscript declare no relationships with any companies, whose products or services may be related to the subject matter of the article.

Statistics and biometry Dr. Renato Cuocolo, one of the authors, kindly provided statistical advice for this manuscript.

Informed consent The respective Local Institutional Review Board for each Institution approved this retrospective study and waived the need for written informed consent.

\section{Methodology \\ - Retrospective \\ - Observational \\ - Multicenter}

Open Access This article is licensed under a Creative Commons Attribution 4.0 International License, which permits use, sharing, adaptation, distribution and reproduction in any medium or format, as long as you give appropriate credit to the original author(s) and the source, provide a link to the Creative Commons licence, and indicate if changes were made. The images or other third party material in this article are included in the article's Creative Commons licence, unless indicated otherwise in a credit line to the material. If material is not included in the article's Creative Commons licence and your intended use is not permitted by statutory regulation or exceeds the permitted use, you will need to obtain permission directly from the copyright holder. To view a copy of this licence, visit http://creativecommons.org/licenses/by/4.0/.

\section{References}

1. Ahdoot M, Wilbur AR, Reese SE et al (2020) MRI-targeted, systematic, and combined biopsy for prostate cancer diagnosis. N Engl J Med 382:917-928. https://doi.org/10.1056/NEJMoa1910038

2. Ahmed HU, El-Shater Bosaily A, Brown LC et al (2017) Diagnostic accuracy of multi-parametric MRI and TRUS biopsy in prostate cancer (PROMIS): a paired validating confirmatory study. Lancet 389:815-822. https://doi.org/10.1016/S01406736(16)32401-1

3. Kasivisvanathan V, Rannikko AS, Borghi M et al (2018) MRItargeted or standard biopsy for prostate-cancer diagnosis. N Engl J Med 378:1767-1777. https://doi.org/10.1056/NEJMoa1801993

4. Park SY, Cho NH, Jung DC, Oh YT (2018) Prostate imagingreporting and data system version 2: beyond prostate cancer detection. Korean J Radiol 19:193. https://doi.org/10.3348/kjr.2018.19. 2.193

5. (2019) EAU Guidelines. Edn. presented at the EAU Annual Congress Barcelona 2019. https://uroweb.org/guideline/prostatecancer. Accessed 13 May 2019

6. de Rooij M, Hamoen EHJ, Witjes JA et al (2016) Accuracy of magnetic resonance imaging for local staging of prostate cancer: a diagnostic meta-analysis. Eur Urol 70:233-245. https://doi.org/10. 1016/j.eururo.2015.07.029

7. Costa DN, Passoni NM, Leyendecker JR et al (2018) Diagnostic utility of a likert scale versus qualitative descriptors and length of capsular contact for determining extraprostatic tumor extension at multiparametric prostate MRI. AJR Am J Roentgenol 210:10661072. https://doi.org/10.2214/AJR.17.18849

8. Freifeld Y, Diaz de Leon A, Xi Y et al (2019) Diagnostic performance of prospectively assigned likert scale scores to determine extraprostatic extension and seminal vesicle invasion with multiparametric MRI of the prostate. AJR Am J Roentgenol 212: 576-581. https://doi.org/10.2214/AJR.18.20320

9. Mehralivand S, Shih JH, Harmon S et al (2019) A grading system for the assessment of risk of extraprostatic extension of prostate cancer at multiparametric MRI. Radiology 290:709-719. https:// doi.org/10.1148/radiol.2018181278

10. Park KJ, Kim M, Kim JK (2020) Extraprostatic tumor extension: comparison of preoperative multiparametric MRI criteria and histopathologic correlation after radical prostatectomy. Radiology 296:87-95. https://doi.org/10.1148/radiol.2020192133

11. Turkbey B, Rosenkrantz AB, Haider MA et al (2019) Prostate imaging reporting and data system version 2.1: 2019 update of prostate imaging reporting and data system version 2 . Eur Urol 76:340-351. https://doi.org/10.1016/j.eururo.2019.02.033

12. Rosenkrantz AB, Shanbhogue AK, Wang A et al (2016) Length of capsular contact for diagnosing extraprostatic extension on prostate MRI: assessment at an optimal threshold. J Magn Reson Imaging 43:990-997. https://doi.org/10.1002/jmri.25040

13. Kim T-H, Woo S, Han S et al (2020) The diagnostic performance of the length of tumor capsular contact on MRI for detecting prostate cancer extraprostatic extension: a systematic review and meta-analysis. Korean J Radiol 21:684. https://doi.org/10.3348/kjr.2019. 0842 
14. Rizzo S, Botta F, Raimondi S et al (2018) Radiomics: the facts and the challenges of image analysis. Eur Radiol Exp 2:36. https://doi. org/10.1186/s41747-018-0068-Z

15. van Timmeren JE, Cester D, Tanadini-Lang S et al (2020) Radiomics in medical imaging - "how-to" guide and critical reflection. Insights Imaging 11:91. https://doi.org/10.1186/s13244-02000887-2

16. Cuocolo R, Cipullo MB, Stanzione A et al (2020) Machine learning for the identification of clinically significant prostate cancer on MRI: a meta-analysis. Eur Radiol. https://doi.org/10.1007/s00330020-07027-w

17. Losnegård A, Reisæter LAR, Halvorsen OJ et al (2020) Magnetic resonance radiomics for prediction of extraprostatic extension in non-favorable intermediate- and high-risk prostate cancer patients. Acta Radiol 028418512090506. https://doi.org/10.1177/ 0284185120905066

18. Xu L, Zhang G, Zhao L et al (2020) Radiomics based on multiparametric magnetic resonance imaging to predict extraprostatic extension of prostate cancer. Front Oncol 10. https://doi.org/10.3389/fonc.2020.00940

19. Krishna S, Lim CS, McInnes MDF et al (2018) Evaluation of MRI for diagnosis of extraprostatic extension in prostate cancer. J Magn Reson Imaging 47:176-185. https://doi.org/10.1002/jmri.25729

20. Ma S, Xie H, Wang H et al (2019) MRI-based radiomics signature for the preoperative prediction of extracapsular extension of prostate cancer. J Magn Reson Imaging 50:1914-1925. https://doi.org/ 10.1002/jmri.26777

21. Stanzione A, Cuocolo R, Cocozza S et al (2019) Detection of extraprostatic extension of cancer on biparametric MRI combining texture analysis and machine learning: preliminary results. Acad Radiol 26:1338-1344. https://doi.org/10.1016/j.acra.2018.12.025

22. Tan PH, Cheng L, Srigley JR et al (2011) International Society of Urological Pathology (ISUP) Consensus conference on handling and staging of radical prostatectomy specimens. Working group 5: surgical margins. Mod Pathol 24:48-57. https://doi.org/10. 1038/modpathol.2010.155

23. Li X, Morgan PS, Ashburner J et al (2016) The first step for neuroimaging data analysis: DICOM to NIfTI conversion. J Neurosci Methods 264:47-56. https://doi.org/10.1016/j.jneumeth.2016.03. 001

24. Yushkevich PA, Gao Y, Gerig G (2016) ITK-SNAP: an interactive tool for semi-automatic segmentation of multi-modality biomedical images. In: 2016 38th Annual International Conference of the IEEE
Engineering in Medicine and Biology Society (EMBC). IEEE, pp $3342-3345$

25. van Griethuysen JJM, Fedorov A, Parmar C et al (2017) Computational radiomics system to decode the radiographic phenotype. Cancer Res 77:e104-e107. https://doi.org/10.1158/00085472.CAN-17-0339

26. Pedregosa F, Varoquaux G, Gramfort A, et al (2012) Scikit-learn: machine learning in Python

27. Eibe F, Hall MA, Witten IH (2016) The Weka Workbench: Online Appendix for "Data Mining: Practical Machine Learning Tools and Techniques," 4th Ed.

28. Koo TK, Li MY (2016) A guideline of selecting and reporting intraclass correlation coefficients for reliability research. J Chiropr Med 15:155-163. https://doi.org/10.1016/j.jcm.2016.02.012

29. Chawla NV, Bowyer KW, Hall LO, Kegelmeyer WP (2002) SMOTE: synthetic minority over-sampling technique. J Artif Intell Res 16:321-357. https://doi.org/10.1613/jair.953

30. (2019) Prostate Imaging-Reporting and Data System Version2.1. In: Am. Coll. Radiol. Web Site. https://www.acr.org/-/media/ACR/ Files/RADS/PI-RADS/PIRADS-V2-1.pdf. Accessed 12 Sep 2020

31. R Core Team (2020) R: a language and environment for statistical computing

32. Sushentsev N, Caglic I, Sala E et al (2020) The effect of capped biparametric magnetic resonance imaging slots on weekly prostate cancer imaging workload. Br J Radiol 93:20190929. https://doi. org $/ 10.1259 /$ bjr.20190929

33. Stanzione A, Ponsiglione A, Cuocolo R et al (2019) Abbreviated protocols versus multiparametric MRI for assessment of extraprostatic extension in prostatic carcinoma: a multireader study. Anticancer Res 39:4449-4454. https://doi.org/10.21873/anticanres. 13617

34. Alderson PO (2020) The quest for generalizability in radiomics. Radiol Artif Intell 2:e200068. https://doi.org/10.1148/ryai. 2020200068

35. Cuocolo R, Caruso M, Perillo T et al (2020) Machine learning in oncology: a clinical appraisal. Cancer Lett. https://doi.org/10.1016/ j.canlet.2020.03.032

36. Choyke PL (2020) A grading system for extraprostatic extension of prostate cancer that we can all agree upon? Radiol Imaging Cancer 2:e190088. https://doi.org/10.1148/rycan.2019190088

Publisher's note Springer Nature remains neutral with regard to jurisdictional claims in published maps and institutional affiliations. 